\title{
3 Research Square \\ Loss of HOXB3 promotes development of hormone receptor negative breast cancer
}

\section{Lizhe Zhu}

the First Affiliated Hospital of Xi'an Jiaotong University https://orcid.org/0000-0002-5507-6323

Shibo Yu

the First Affiliated Hospital of Xi'an Jiaotong University

\section{Siyuan Jiang}

the First Affiliated Hospital of Xi'an Jiaotong University

\section{Guanqun Ge}

the First Affiliated Hospital of Xi'an Jiaotong Univerisity

Yu Yan

the First Affiliated Hospital of Xi'an Jiaotong University

\section{Yuhui Zhou}

the First Affiliated Hospital of Xi'an Jiaotong University

\section{Ligang Niu}

the First Affiliated Hospital of Xi'an Jiaotong University

Jianjun He

the First Affiliated Hospital of Xi'an Jiaotong University

Yu Ren ( $\nabla$ renyyyyy@126.com )

the First Affiliated Hospital of Xi'an Jiaotong University

Bin Wang ( $\square$ february111@163.com )

the First Affiliated Hospital of Xi'an Jiaotong University https://orcid.org/0000-0002-2662-1986

\section{Primary research}

Keywords: HOXB3, hormone receptor negative, breast cancer, bioinformatic, prognosis

Posted Date: April 22nd, 2020

DOI: https://doi.org/10.21203/rs.3.rs-23549/v1

License: (1) This work is licensed under a Creative Commons Attribution 4.0 International License.

Read Full License 


\section{Abstract}

\section{Background}

The homobox (HOX) gene family as a transcription factor encoding a specific nuclear protein is essential for embryonic development, differentiation, and homeostasis. The role of HOXB3 protein varies in different tumors. This study aims to explore the role of the HOXB3 gene in breast cancer.

Method

Differentiated expressed genes were screened by analyzing metastatic breast cancer gene chip data in TCGA and GEO database. The function of selected HOXB3 gene was also analyzed by GEPIA, KaplanMeier Plotter, Breast Cancer Gene-Expression Miner and metascape. Molecular biology methods such as qRT-PCR, western blot and IF was used to verify bio-informatics findings.

Results

Both bio-informatics analyses and western blot showed that HOXB3 was lost in breast cancer compared to normal breast tissue. Survival analysis also showed that lower expression of HOXB3 was associated with poor prognosis. Bio-informatics analyses further showed that HOXB3 was positively correlated with hormone receptors. qRT-PCR, immunofluorescence and western blot also confirmed that HOXB3 had the highest expression in the immortalized breast epithelial cell line MCF-10A, lower in luminal breast cancer cell line T47D and the lowest in triple negative breast cancer (TNBC) cell line MDA-MB-231. Metascape for $\mathrm{GO}$ analysis of GEO data provided possible mechanism that HOXB3 could positively regulate cell adhesion, inhibit cell proliferation and activate immune response in breast cancer, and considered that HOXB3 might cause cell malignant transformation through the above pathways.

\section{Conclusion}

In summary, HOXB3 expression was decreased in breast cancer, especially in hormone receptor-negative breast cancer. The lower expression of HOXB3 was associated with poor prognosis. It might become a new biomarker to predict prognosis of breast cancer.

\section{Background}

The incidence of breast cancer among women in China has increased year by year recently. According to statistics, the annual incidence of breast cancer in women in China is about 208000 and the total crude incidence rate is $32.43 / 100000$. This incidence accounts for $16.20 \%$ of all female cancer cases and becomes the first rank among all cancer incidences [1]. Study has shown that the rapid growth rate in Chinese female has exceeded the world average growth rate of $2 \%$ [2]. At the same time, breast cancer has become one of the leading causes of death among young women in China [3]. In western countries, breast cancer is the second most common cancer among American women after skin cancer, and it is the second leading cause of cancer death in the United States after lung cancer [4]. Among women, the most 
common cancer diagnosed between the ages of 30 and 59 is breast cancer, and among women under the age of 45 , breast cancer is the leading cause of death. Breast cancer as a malignant tumor with complicated biological behavior in the female reproductive system has seriously harmed women's life and health.

The development of genomics engineering has opened up new fields for studies of the pathogenesis of breast cancer, especially for the genes involved in their growth and their pathways. Among them, the homobox (HOX) gene family, as a family of regulatory genes, contains a homologous domain transcription factor, which can encode a specific nuclear protein as a transcription factor, which is crucial for embryonic development, differentiation, and homeostasis. In recent years, many studies have shown that the imbalance of HOX gene is inextricably associate with the occurrence and invasion of various tumors. Laixi Bi et al found that a miR-375-HOXB3-CDCA3/DNMT3B regulatory pathway containing the HOXB3 gene was involved in the development of acute myeloid leukemia [5]. Dejun Yang et al found that down-regulation microRNA-375 could inhibit the proliferation, migration and chemo-sensitivity of pancreatic cancer through the combination with HOXB3 [6]. Jing Chen [7] and others had shown that HOXB3 could promote the progression of prostate cancer cells by activating CDCA3. Katherine R. Miller [8] and others demonstrated that HOXA4/HOXB3 gene expression could be used as a recurrence marker after primary cytoreductive surgery and first-line adjuvant chemotherapy for high-grade serous ovarian cancer. Thus, the abnormal expression of the HOX gene family had been reported in a variety of malignant tumors with abnormal development, suggesting that changes in the HOX gene might have important implications for tumorigenesis and inhibition.

At present, there were few studies on the HOXB3 gene in breast cancer. Therefore, this study screened differentially expressed genes of breast cancer metastasis by analyzing breast cancer metastasis-related gene chip data in databases such as TCGA and gene chip public database (GEO). Metascape, and Breast Cancer Gene-Expression Miner and other software were used to analyze data. qRT-PCR, western blot, Immunofluorescence and other molecular biological methods were applied to verify clinical findings and analyze its association with breast cancer immunophenotyping and prognostic values. So our data can comprehensively provide insights and ideas for further exploring the molecular metastasis of breast cancer metastasis.

\section{Method}

\section{GEPIA}

GEPIA (Gene Expression Profiling Interactive Analysis, http://gepia.cancer-pku.cn/) [9] is a web which served not only for cancer and normal gene expression profiling, but also for interactive analyses. This web tool can analyze the RNA sequencing expression data of approximately 9736 tumors and 8587 normal samples. All samples are excavated from TCGA and GTEx projects by using a standard processing pipeline. It provides different kinds of functions including tumor vs normal differential expression analysis, patients survival analysis, similar gene detection and etc. The differential expression 
of HOXB3 in normal breast tissue and breast cancer was detected by GEPIA. A log P-value $<0.01$ was considered statistically significant.

\section{Kaplan-Meier Plotter database}

The Kaplan-Meier Plotter $[10,11]$ is a tool which based on meta-analysis biomarker assessment. This web tool can assess the survival of multiple tumors such as breast, ovarian, lung and etc. The gene expression data and relapse free survival information are downloaded from TCGA, GEO and EGA. Handled by a PostgreSQL server, this database is able to integrate clinical data and gene expression simultaneously. We used this database to analyze the prognostic value of HOXB3 mRNA expression in all breast cancers. A log P-value $<0.01$ was considered statistically significant.

\section{Breast Cancer Gene-Expression Miner (bc-GenExMiner) v4.3}

Bc-GenExMiner v4.3 [12,13] is a statistical mining tool which including a large number of published annotated breast cancer transcriptomic data with both RNA-sequence and DNA microarrays. It can perform the statistical analysis of prognosis, expression and correlation. The correlation between $\mathrm{HOXB3}$ and ESR1, ESR2, PGR, ERBB2 and MKI67 were analyzed by using Bc-GenExMiner v4.3. The relationship between HOXB3 and the clinicopathologic parameters (ER, PR, HER2, SBR, molecular subtypes) of breast cancer were also analyzed on this online tool.

\section{GEO database and Metascape}

We downloaded the original dataset for comparing the gene expression profile between breast cancer and normal breast tissue from the NCBI GEO database (the accession number was GSE27447). Later, we used GEO2R and Metascape to perform GO and KEGG pathway analysis on target gene HOXB3. Metascape (http://metascape.org)[14] was a gene annotation and analysis resource which helped us to do the gene enrichment analysis and found the relevantly important signal pathways.

\section{Cell culture and reagents}

Human breast cancer cell MDA-MB-231 and T47D were maintained in DMEM High Glucose and RPMI 1640 medium (Gibco; Thermo Fisher Scientific, Inc., Waltham, MA, USA) respectively supplied with 10\% fetal bovine serum (FBS; Gibco; Thermo Fisher Scientific, Inc.) at $37^{\circ} \mathrm{C}$ with $5 \% \mathrm{CO}_{2}$. The MCF- $10 \mathrm{~A}$ cells were cultured in DMEM/F12 (1:1) medium (Gibco; Thermo Fisher Scientific, Inc., Waltham, MA, USA) supplemented with $100 \mathrm{ng} / \mathrm{ml}$ cholera toxin (Sigma, St. Louis, MO, USA), $20 \mathrm{ng} / \mathrm{ml}$ epidermal growth

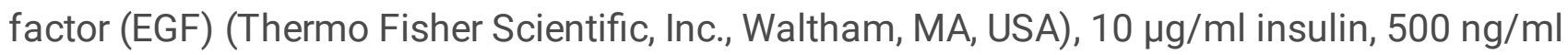
hydrocortisone and $5 \%$ heat-inactivated horse serum (all from Sigma) at $37^{\circ} \mathrm{C}$ with $5 \% \mathrm{CO}_{2}$. Old cell culture medium was replaced with fresh medium every alternate day.

\section{Real-time quantitative polymerase chain reaction (qRT-PCR)}


Total RNA was extracted with RNeasy mini kit (Qiagen, Valencia, CA) according to the manufacturer's protocol. The concentration and purity of all RNA samples were determined at an absorbance ratio of 260/280 nm. Total 1ug RNA was reverse-transcribed using iScript ${ }^{\mathrm{TM}}{ }^{\mathrm{CDNA}}$ Synthesis kit from Bio-Rad (Hercules, CA). Real-time PCR analysis was set up with SYBR Green qPCR Supermix kit (Invitrogen, Carlsbad, CA) and carried out in the iCycler thermal cycler. The relative level of mRNA expression of gene was determined by normalizing with GAPDH. Primers for HOXB3: forward-5' TGCTGCTGGGAGACTCGTAA 3'; reverse-5' GCATCCCCTTGCAGCTAAAC 3'; GAPDH: forward-5' AAGGCTGTGGGCAAGGTCATC 3'; reverse-5' GCGTCAAAGGTGGAGGAGTGG 3'.

\section{Western blot}

The expression level of HOXB3 in different molecular types of breast tissues was analyzed by western blot. Human breast tissues were minced and transformed into homogenizer, and later lysed in RIPA buffer containing phosphatase and protease inhibitor by using ultrasonic crusher (SONICS \& MATERIALS INC. USA). Then, the breast tissue homogenate was kept on ice for $20 \mathrm{~min}$ and transferred to centrifuge tube for centrifuge at $4^{\circ} \mathrm{C}, 20 \mathrm{~min}$. The upper supernatant of breast tissue proteins were collected and detected by BCA Protein Assay Kit (Pierce; Thermo Fisher Scientific, Inc.). 30 ug of protein was separated on 10\% SDS-PAGE gels and transferred to polyvinylidene fluoride (PVDF) membranes (Merck Millipore, Billerica, MA, USA). The membranes were blocked by $5 \%$ nonfat milk in TBST in order to block the non-specific binding. And subsequently incubated with primary antibody (HOXB3: cat no. ITA9318; G-Biosciences, Inc.; dilution, 1:2,000; GAPDH: cat no. 5174; Cell Signaling Technology, Inc.; dilution, 1:1,000) overnight at $4{ }^{\circ} \mathrm{C}$. All samples were incubated with anti-horseradish peroxidase-linked IgG secondary antibody (cat no. 7074; Cell Signaling Technology, Inc.; dilution, 1:2,000) at room temperature for $2 \mathrm{~h}$ and detected via chemiluminescence detection system (version 3.0; Bio-Rad Laboratories, Inc., Hercules, CA, USA).

\section{Immunofluorescence}

Cells were washed with PBS and were fixed with $4 \%$ paraformaldehyde. Subsequently $0.5 \%$ Triton X-100 was used to perforate cells and $5 \%$ bovine serum albumin (BSA) was used to block cells. Cells were then incubated with primary antibody (HOXB3: cat no. ITA9318; G-Biosciences, Inc.) at $4^{\circ} \mathrm{C}$ overnight and Alexa Fluor 647-conjugated secondary antibodies, followed by nuclear staining with $5 \mathrm{ug} / \mathrm{ml}$ 4',6diamidino-2-phenylindole (DAPI, Invitrogen). Signals were examined using a fluorescence microscope.

\section{Results}

\section{Decreased expression of HOXB3 in breast cancer patients}

HOXB3 expression was analyzed in breast cancer patients compared with health women in the GEPIA database. Figure 1A showed that the expression of HOXB3 gene in breast cancer patients was significantly lower than that in normal subjects, and the difference was statistically significant. We further tested the HOXB3 gene expression in proteins extracted from human breast tissues. Figure 1B and 1C also showed that the HOXB3 was down-regulated in breast cancer tissues compared with normal ones. 
These data suggested that HOXB3 might decreased in breast cancer patients and might play critical roles in breast cancer cell carcinogenesis.

\section{Prognostic values of HOXB3 mRNA expression in all breast cancers}

Figure 2 showed the significant RFS curves associated with HOXB3 gene. The Kaplan-Meier Plotter was used to examine the prognostic values of HOXB3 mRNA expression level in all breast cancers (Figure 2). Figure 2 revealed that a low mRNA expression of HOXB3 was associated with a worse prognosis of RFS (Figure 2A, HR= 0.83, 95\% Cl: 0.74-0.92, $\mathrm{P}=0.00076$; Figure 2B, HR=0.77, 95\% Cl: 0.66-0.9, $\mathrm{P}=0.00079$ ). The results were the same with the data that lower expression of HOXB3 existed in breast cancer patients.

\section{The correlation between HOXB3 and hormone receptors}

Figure 3 showed that HOXB3 was closely correlated with hormone receptors related genes, such as ESR1, ESR2, PGR, ERBB2 and MKI67. The Bc-GenExMiner v4.3 was used to analyze the relationship between HOXB3 and those clinicopathologic parameters of breast cancer. Result showed that HOXB3 was positively correlated with ESR1, PGR and ERBB2 (ESR1: $r=0.28,<P 0.0001$; PGR: $r=0.23, P<0.0001$; ERBB2: $r=0.10, P<0.0001$ ) (Figure 3). Figure 4A-D also showd that a higher mRNA level of HOXB3 was associated with positive expression of hormone receptor (ER and PR), no matter in DNA microarrays or in RNAsequence level. Furthermore, for the Scarff-Bloom-Richardson (SBR) grade criterion shown in Figure 4E and $4 \mathrm{~F}$, a higher mRNA level of HOXB3 was associated with a lower SBR grade in both DNA microarrays and RNA-sequence. All of the pairwise comparisons in the SBR criteria were statistically significant $(P<0.01)$.

\section{HOXB3 mRNA expression in different molecular subtypes in breast cancer}

Figure 5 showed that both in DNA microarrays and RNA-sequence levels, a higher mRNA level of HOXB3 was correlated with normal-breast like tissues or luminal subtypes breast cancer. A relatively lower mRNA level of HOXB3 was correlated with HER2 or basal-like breast cancer. We further detected the specific gene expression by molecular biology method. By qRT-PCR, we confirmed that HOXB3 had the highest expression in the immortalized breast epithelial cell line MCF-10A, lower in luminal breast cancer cell line T47D and the lowest in triple negative breast cancer (TNBC) cell line MDA-MB-231 (Figure 6A). Besides, western blot showed that HOXB3 was highly expressed in normal breast tissues, moderately expressed in luminal breast cancer, and lowly expressed in TNBC (Figure 6B and 6C). Immunofluorescence showed that the HOXB3 gene was nuclear localized, and the fluorescence intensity was strong in the luminal breast cancer cell line T47D, and the fluorescence intensity was weak in the TNBC cell line MDA-MB-231 (Figure 6D). It was suggested that the HOXB3 gene might be expressed less in aggressive breast cancer subtypes.

\section{Metascape for GO analysis of possible mechanism of HOXB3 in breast cancer}

By analyzing the NCBI GEO database, we found out the differentially expressed gene HOXB3. Later, GEO2R and Metascape was used to perform GO and KEGG pathway analysis on our target gene HOXB3. 
The bioinformatic analysis revealed the signaling pathways related to the HOXB3. As shown in figure 7, the related signaling pathways involved positive regulation of cell matrix adhesion, actin cytoskeletal remodeling, chemotaxis regulation, and classical Wnt signaling pathways. Those possible mechanism suggested that HOXB3 could positively regulate cell adhesion, inhibit cell proliferation and activate immune response in breast cancer, and considered that HOXB3 might cause cell malignant transformation through the above pathways.

\section{Discussion}

Some researchers believe that the occurrence of tumors reproduces the process of early embryo development and embryonic aberration [15]. Researches on embryo development related genes and tumorigenesis has become another research hotspot in molecular oncology, and the homeobox gene is one of them [16-18]. HOXB3 has different effects in promoting cancer or suppressing cancer in different types of tumors. Hui Fu [19] and others found that HOXB3 is a target of miR-375 in MCF-7 cells. Moreover, HOXB3 could not only significantly promote EMT in MCF-7 cells, but also promote the formation of CSC phenotype and tamoxifen resistance. Qiaoyan Li et al [20] found that miR-7 and miR-218 can regulate breast cancer suppressor genes RASSF1 A and Claudin-6 through targeting HOXB3. Although both studies considered HOXB3 to be an oncogene, they were all performed in cell model. In fact, cell model had great limitations and was affected by culture conditions and was unable to reflect the real situation of breast cancer patients. Our study was to analyze a large number of data in the international common database (TCGA and GEO database) and to verify the patient specimens by molecular biological methods. The mRNA and protein levels of HOXB3 were significantly lower in breast cancer tissues compared to normal ones. This result was consistent with Svingen's team [21]. Their team verified the expression of normal breast tissue, normal breast cell line SVCT, breast cancer cell line MCF-7 and MDA-MB-231, and found that the expression of HOXB3 in breast cancer cells was much lower than that in normal breast tissue and normal breast cell line SVCT. Moreover, the HOXB3 expression of the melanoma cell lines MM96L, MM418c1 and MM48C5 was also much lower than that of human skin fibroblast HSF cells. As we showed in supplementary figure 1, HOXB3 was reduced to varying degrees in the expression profiles of multiple cancer species. This indicated that the loss of HOXB3 was prevalent in many cancer species. HOXB3 might be a tumor suppressor gene in these specific cancers and breast cancer was one of them.

Since the expression of HOXB3 was lost in breast cancer, whether low expression of HOXB3 indicated a poor prognosis was still unknown. Then we verified the survival curve and found that the low expression of HOXB3 gene was indeed associated with poor prognosis in breast cancer patients. We used Metascape to perform GO analysis on GEO data, the results showed that HOXB3 could positively regulate cell adhesion, inhibit cell proliferation and activate immune response in breast cancer. Consistent with the above results, TCGA data also suggested a negative correlation between HOXB3 and the cell proliferation index Ki67. Therefore, we suspected that the absence of HOXB3 might lead to cell malignant transformation through the above pathways. 
The molecular subtypes of breast cancer is a unnegligible variable in breast cancer research. Different molecular subtypes of breast cancer showed different malignant behaviors and required different treatments. Our study also analyzed HOXB3 in different subtypes of breast cancer. Our result suggested that there was a significant positive correlation between HOXB3 and hormone receptors ER and PR, but no significant correlation with HER2 expression. Clinically, hormone receptor-negative subtype indecates highe malignancy and worse prognosis, and the lower the expression of hormone receptor is accompanied with the lower expression of HOXB3. This principle was consistent with the findings of lower malignancy correlated with lower expression of HOXB3. Pathological evaluation of breast cancer is not only molecular classification but also SBR pathological grade. The higher grade, the higher malignancy. Consistently, the higher SBR pathological grade was accompanied with the lower HOXB3 expression. These results all prove that HOXB3 may be a tumor suppressor gene, which is consistent with the previous results.

\section{Conclusion}

In summary, we found that HOXB3 expression was decrease in breast cancer by big data analysis and molecular biology experiments. Especially in hormone receptor-negative breast cancer, low expression of HOXB3 was associated with poor prognosis. Identifying people at risk with molecular characteristics may help to develop active surveillance, adjuvant therapy, clinical trial considerations, or alternative treatment intervention decisions for high-risk patients. Especially for patients with negative hormone receptors, whether HOXB3 expression can be used as a prognostic biomarker for breast cancer patients needs further validation in prospective studies.

\section{Abbreviations}

Homobox 3 (HOXB3), Gene Expression Omnibus(GEO), The Cancer Genome Atlas(TCGA), Gene Expression Profiling and Interactive Analyses (GEPIA), Scarff-Bloom-Richardson (SBR), triple negative breast cancer (TNBC).

\section{Declarations}

\section{Ethics approval and consent to participate}

The human breast cancer tissues used in this study have already got consent from the Ethics Committee of the First Affiliated Hospital of Xi'an Jiaotong University, other data in this study were all from the online database, which does not require ethical approval.

\section{Consent for publication}

Not applicable

\section{Availability of data and materials}


The datasets [GSE27447] for this study can be found in the [GEO database] [https://www.ncbi.nlm.nih.gov/geo/query/acc.cgi?acc=GSE27447]. Other publicly available datasets could be found in the following websites: http://gepia.cancer-pku.cn/, https://kmplot.com/analysis/, http://bcgenex.centregauducheau.fr/BC-GEM/GEM-Accueil.php?js=1, http://metascape.org.

\section{Competing interests}

The authors declare that they have no competing interests.

\section{Funding}

The National Natural Science Foundation of China (NSFC 81702633 to Bin Wang).

\section{Authors' contributions}

LZ-Z: Collection and/or assembly of data, data analysis and interpretation, manuscript writing, methodology and software. SB-Y: Collection and/or assembly of data, data analysis and interpretation, manuscript writing and editing. SY-J: Data analysis, manuscript writing and interpretation. GQ-G: Manuscript writing and project administration. Y-Y: Manuscript writing and project administration. YH-Z: Manuscript writing. LG-N: Manuscript writing. JJ-H: Conception/design. Y-R: Conception/design, supervision and editing. B-W: Conception/design, supervision and editing. All authors: Article writing and final approval of article.

\section{Acknowledgments}

Thanks to the experimental platform support of the translational medicine center of the First Affiliated Hospital of Xi'an Jiaotong University. And We also showed our special thanks to Kaplan-Meier Plotter, GEPIA, 2.3 Breast Cancer Gene-Expression Miner (bc-GenExMiner) v4.3, GEO and Metascape for their open access databases.

\section{References}

[1] Zeng H, Zheng R, Zhang S, Zou X, Chen W. Female breast cancer statistics of 2010 in China: estimates based on data from 145 population-based cancer registries. J Thorac Dis. 2014;6(5):466-470.

[2] Shen SJ, Sun Q. Current Status and Suitable Mode Evaluation of Breast Carcinoma Screening in Chinese Women. Medical Journal of Peking Union Medical College Hospital.2018;9(04):298-302.

[3] National Bureau of Statistics of China. China Statistical Yearbook, 2010. Beijing, China: China Statistics Press; 2010.

[4] DeSantis CE, Ma J, Goding Sauer A, Newman LA, Jemal A. Breast cancer statistics, 2017, racial disparity in mortality by state. CA Cancer J Clin. 2017;67(6):439-448. 
[5] Bi L, Zhou B, Li H, et al. A novel miR-375-HOXB3-CDCA3/DNMT3B regulatory circuitry contributes to leukemogenesis in acute myeloid leukemia. BMC Cancer. 2018;18(1):182.

[6] Yang D, Yan R, Zhang X, et al. Deregulation of MicroRNA-375 inhibits cancer proliferation migration and chemosensitivity in pancreatic cancer through the association of HOXB3. Am J Transl Res. 2016;8(3):1551-1559.

[7] Chen J, Zhu S, Jiang N, Shang Z, Quan C, Niu Y. HoxB3 promotes prostate cancer cell progression by transactivating CDCA3. Cancer Lett. 2013;330(2):217-224.

[8] Miller KR, Patel JN, Zhang Q, et al. HOXA4/HOXB3 gene expression signature as a biomarker of recurrence in patients with high-grade serous ovarian cancer following primary cytoreductive surgery and first-line adjuvant chemotherapy. Gynecol Oncol. 2018;149(1):155-162.

[9] Tang Z, Li C, Kang B, Gao G, Li C, Zhang Z. GEPIA: a web server for cancer and normal gene expression profiling and interactive analyses. Nucleic Acids Res. 2017;45(W1): W98-W102.

[10] Lánczky A, Nagy Á, Bottai G, et al. miRpower: a web-tool to validate survival-associated miRNAs utilizing expression data from 2178 breast cancer patients. Breast Cancer Res Treat. 2016;160(3):439446.

[11] Györffy B, Lanczky A, Eklund AC, et al. An online survival analysis tool to rapidly assess the effect of 22,277 genes on breast cancer prognosis using microarray data of 1,809 patients. Breast Cancer Res Treat. 2010;123(3):725-731.

[12] Jézéquel $P$, Campone $M$, Gouraud W, et al. bc-GenExMiner: an easy-to-use online platform for gene prognostic analyses in breast cancer. Breast Cancer Res Treat. 2012;131(3):765-775.

[13] Jézéquel P, Frénel JS, Campion L, et al. bc-GenExMiner 3.0: new mining module computes breast cancer gene expression correlation analyses. Database (Oxford). 2013;2013:bas060. .

[14] Zhou Y, Zhou B, Pache L, et al. Metascape provides a biologist-oriented resource for the analysis of systems-level datasets. Nat Commun. 2019;10(1):1523.

[15] Shah N, Sukumar S. The Hox genes and their roles in oncogenesis. Nat Rev Cancer. 2010;10(5):361371.

[16] Daftary GS, Taylor HS. Endocrine regulation of HOX genes. Endocr Rev. 2006;27(4):331-355.

[17] Chen F, Capecchi MR. Paralogous mouse Hox genes, Hoxa9, Hoxb9, and Hoxd9, function together to control development of the mammary gland in response to pregnancy. Proc Natl Acad Sci U S A. 1999;96(2):541-546. 
[18] Kar SP, Tyrer JP, Li Q, et al. Network-Based Integration of GWAS and Gene Expression Identifies a HOX-Centric Network Associated with Serous Ovarian Cancer Risk. Cancer Epidemiol Biomarkers Prev. 2015;24(10):1574-1584.

[19] Fu H, Fu L, Xie C, et al. miR-375 inhibits cancer stem cell phenotype and tamoxifen resistance by degrading HOXB3 in human ER-positive breast cancer. Oncol Rep. 2017;37(2):1093-1099.

[20] Li Q, Zhu F, Chen P. miR-7 and miR-218 epigenetically control tumor suppressor genes RASSF1A and Claudin-6 by targeting HoxB3 in breast cancer. Biochem Biophys Res Commun. 2012;424(1):28-33.

[21] Svingen T, Tonissen KF. Altered HOX gene expression in human skin and breast cancer cells. Cancer Biol Ther. 2003;2(5):518-523.

\section{Supplemental Figure Legend}

Supplementary Figure 1 HOXB3 expression in different cancer types in GEPIA.

\section{Figures}


A

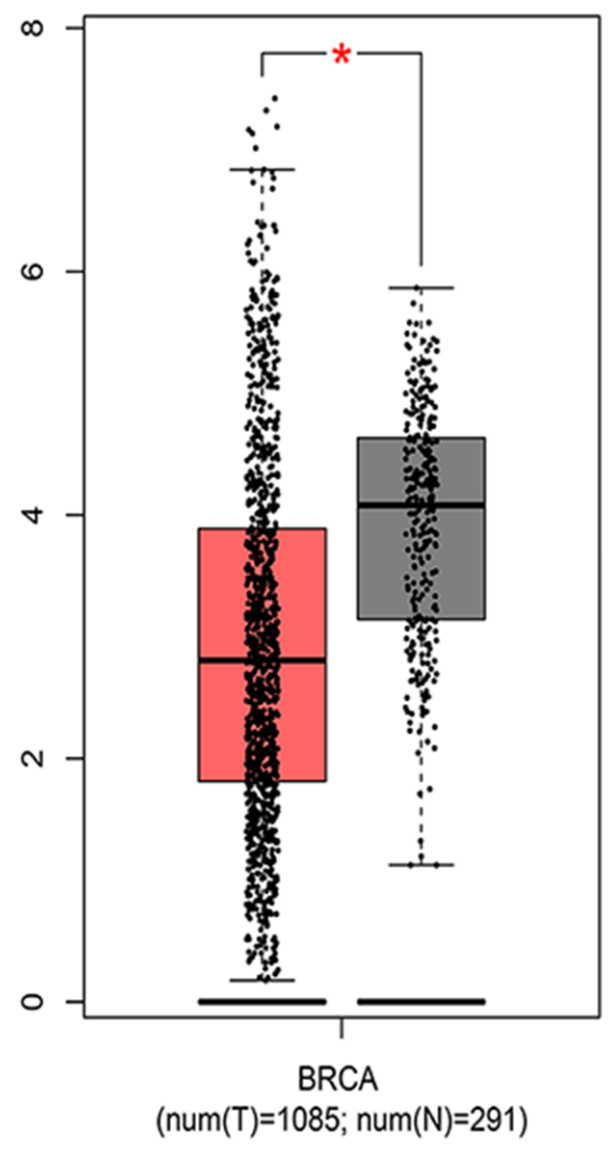

B

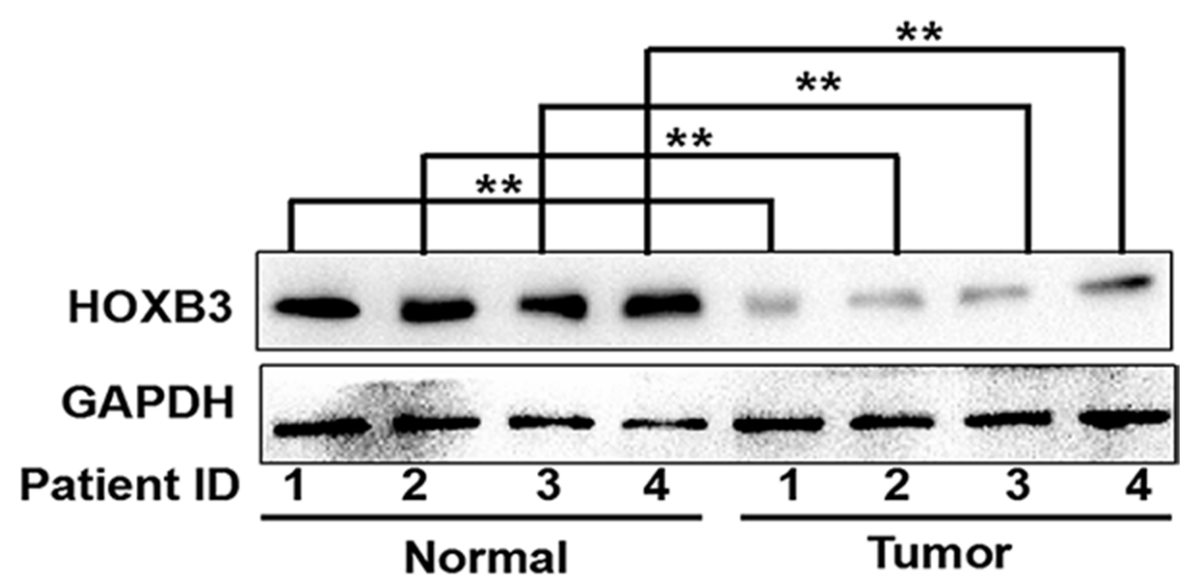

C

HOXB3

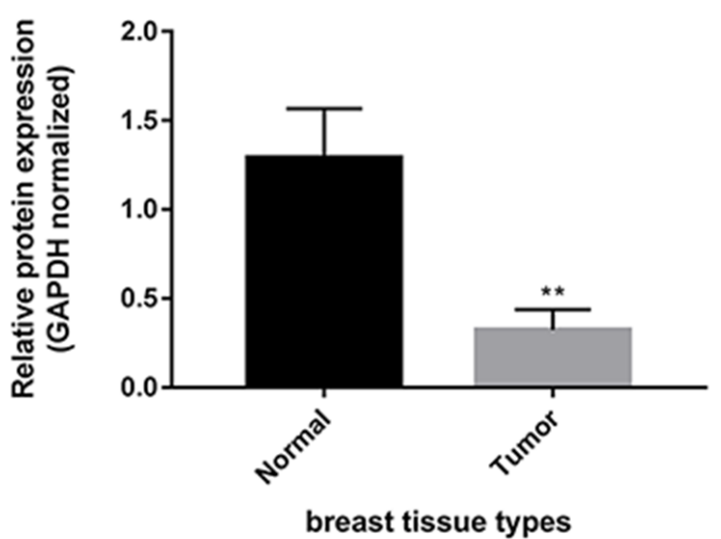

Figure 1

The HOXB3 expression in breast cancer patients compared with normal people. (A) Box plot of HOXB3 expression in breast cancer patients compared with normal people in GEPIA database. (B) The western blot of HOXB3 expression in human breast tissues compared with normal ones. (C) The relative protein expression of HOXB3 (GAPDH normalized) of western blot. $\mathrm{P}<0.05$ was considered statistically

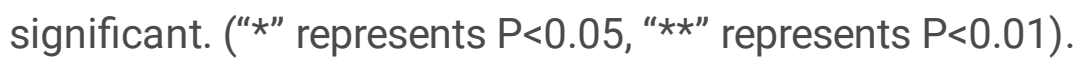


A

208414_s_at

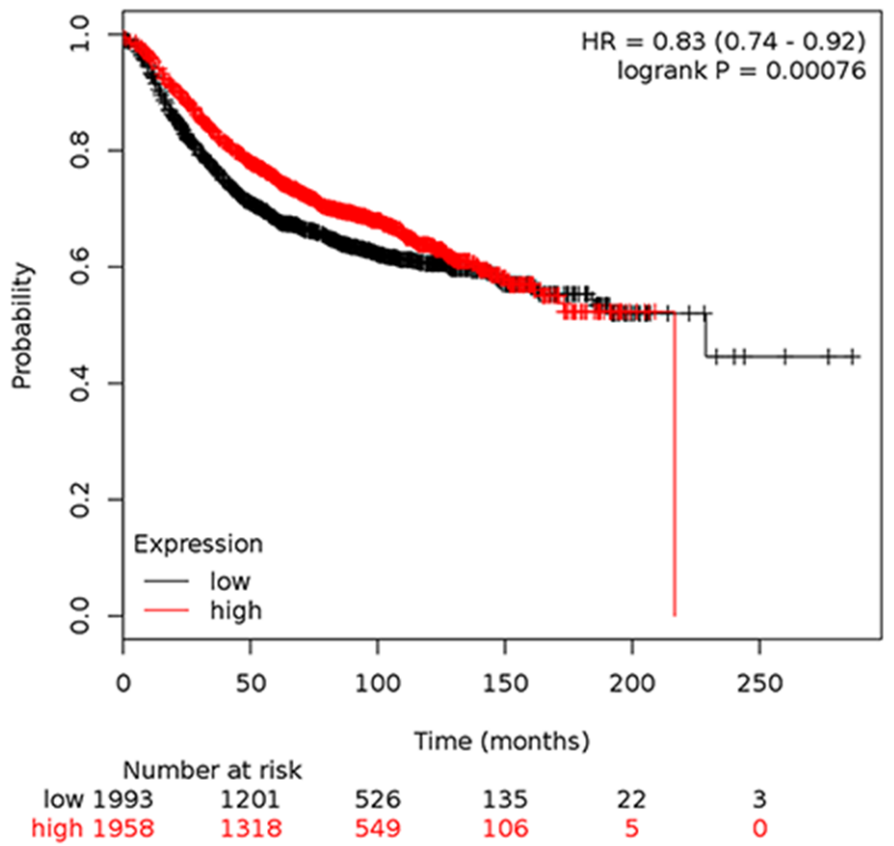

B

228904_at

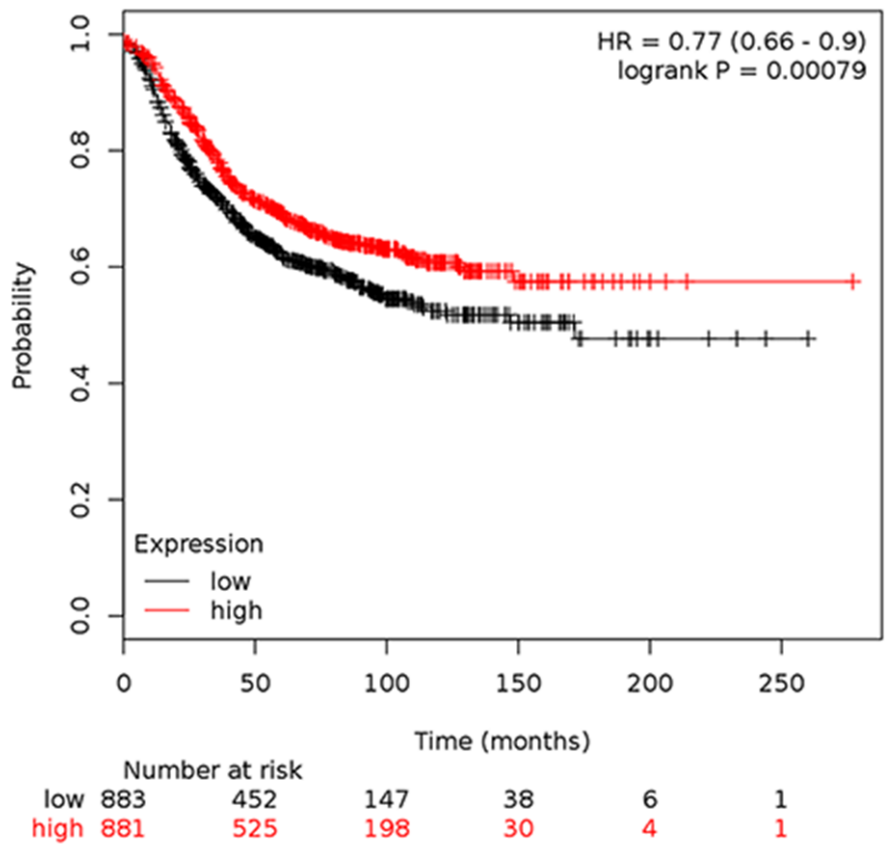

Figure 2

Survival analyses of HOXB3 in breast cancer (RFS in Kaplan-Meier Plotter).

A

$\mathrm{D}$
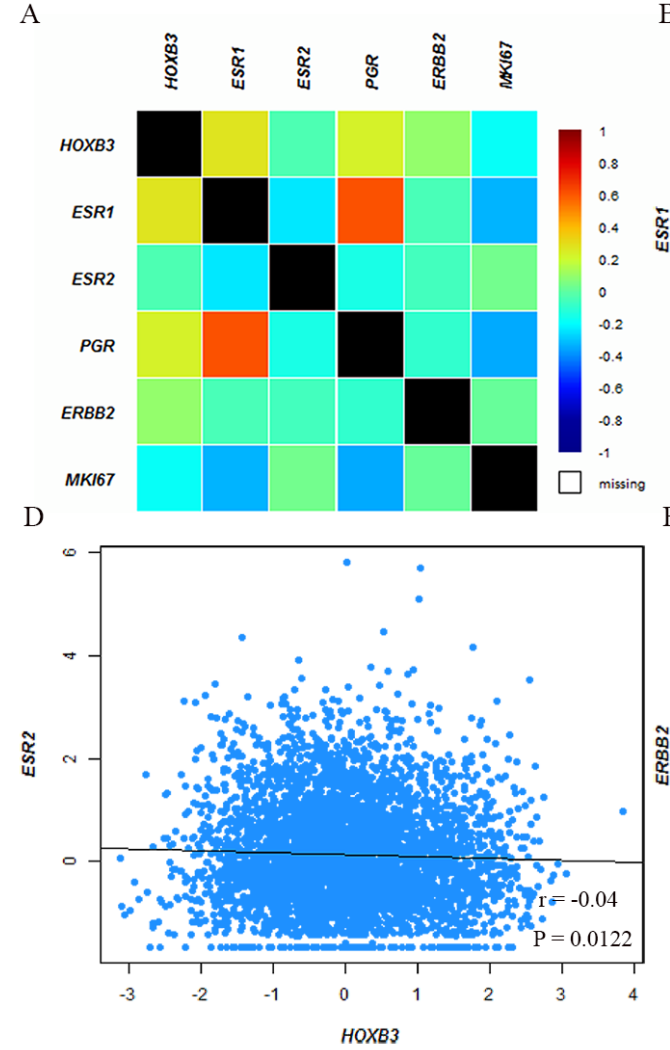

B

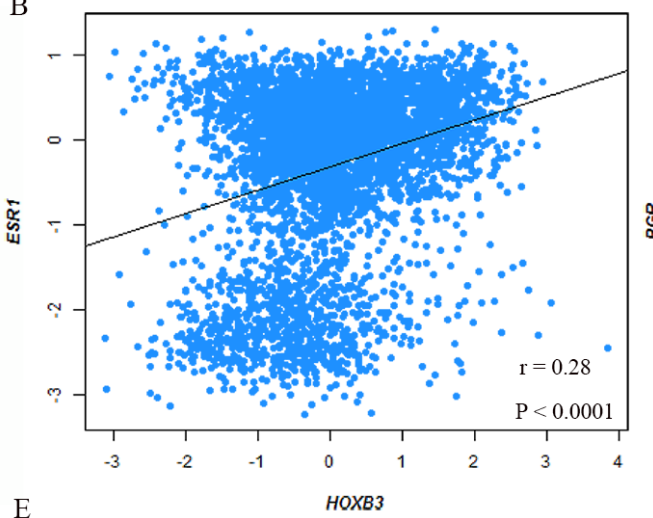

E

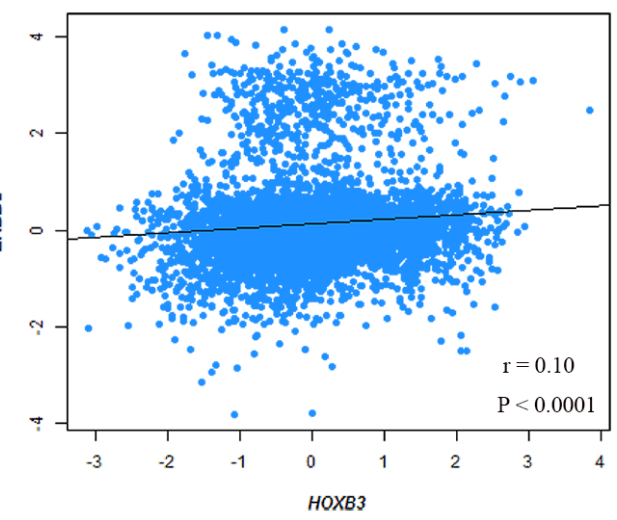

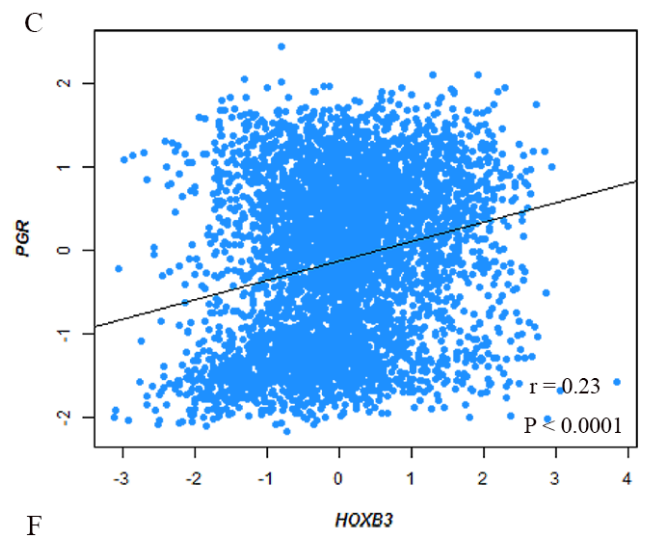

$\mathrm{F}$

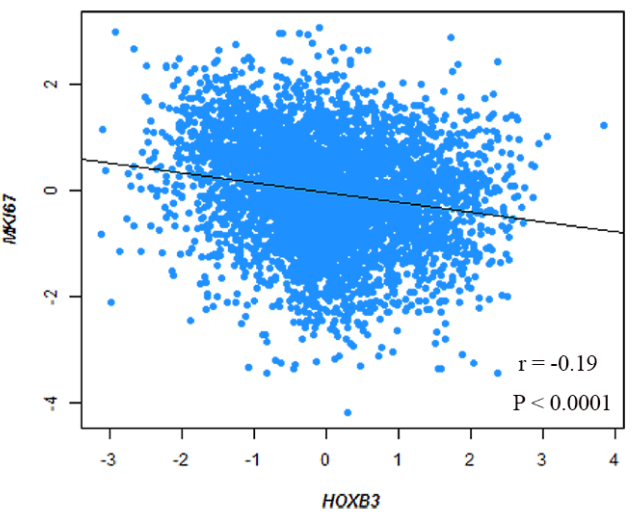

Figure 3 
The correlation between HOXB3 and clinicopathologic parameters of breast cancer. (A) The total correlation heatmap of HOXB3 expression with clinicopathologic parameters. (B) The correlation between HOXB3 and ESR1. (C) The correlation between HOXB3 and PGR. (D) The correlation between HOXB3 and ESR2. (E) The correlation between HOXB3 and ERBB2. (F) The correlation between HOXB3 and MKI67. $\mathrm{P}<0.05$ was considered statistically significant.
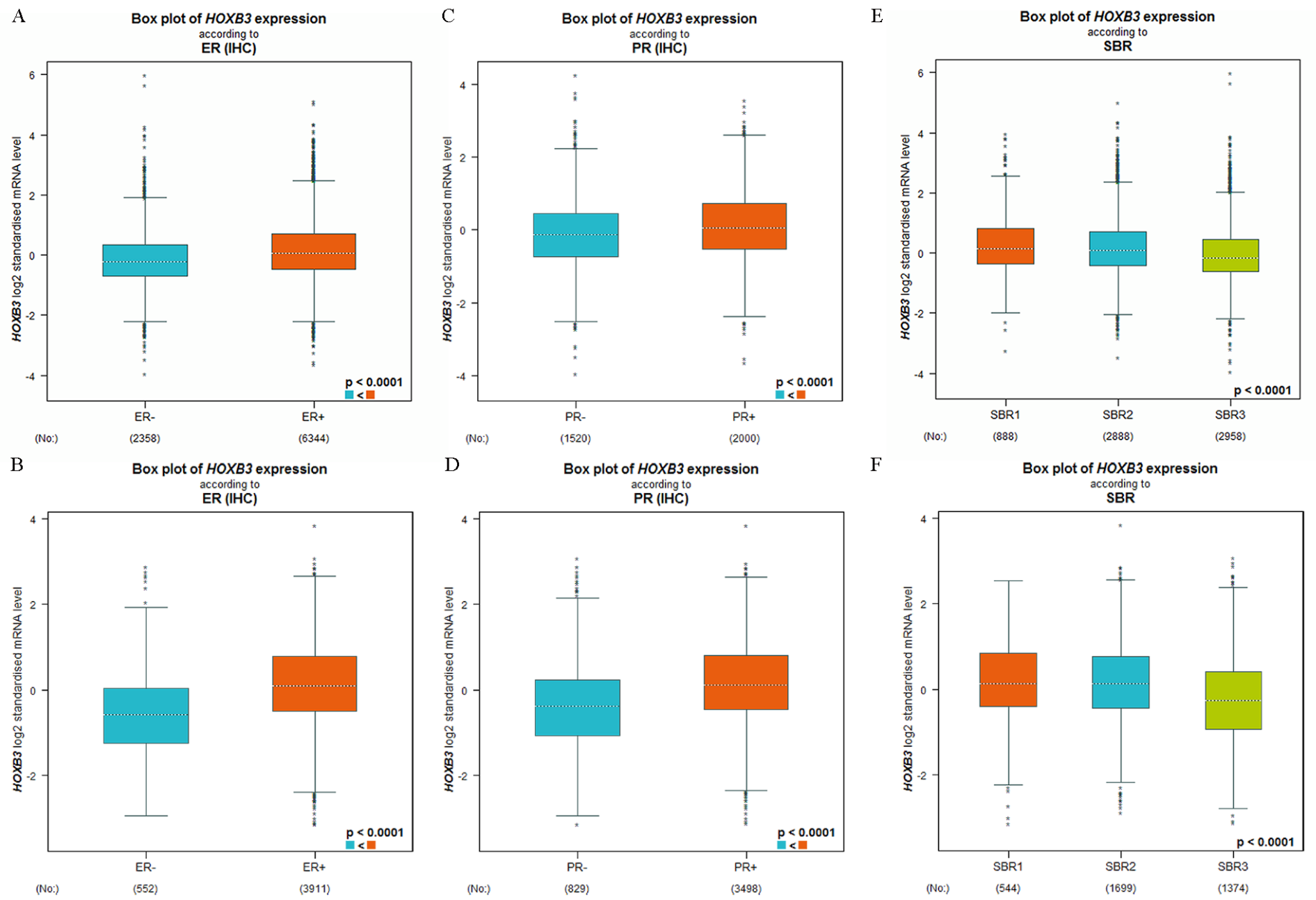

Figure 4

The relationship between HOXB3 and ER, PR and SBR criteria. (A, C, E) Box plots of HOXB3 expression according to ER, PR and SBR (in DNA microarrays' level). (B, D, F) Box plots of HOXB3 expression according to $E R, P R$ and SBR (in RNA-sequence level). Global significant differences between groups were assessed by Welch's test, and $\mathrm{P}<0.05$ was considered statistically significant. 

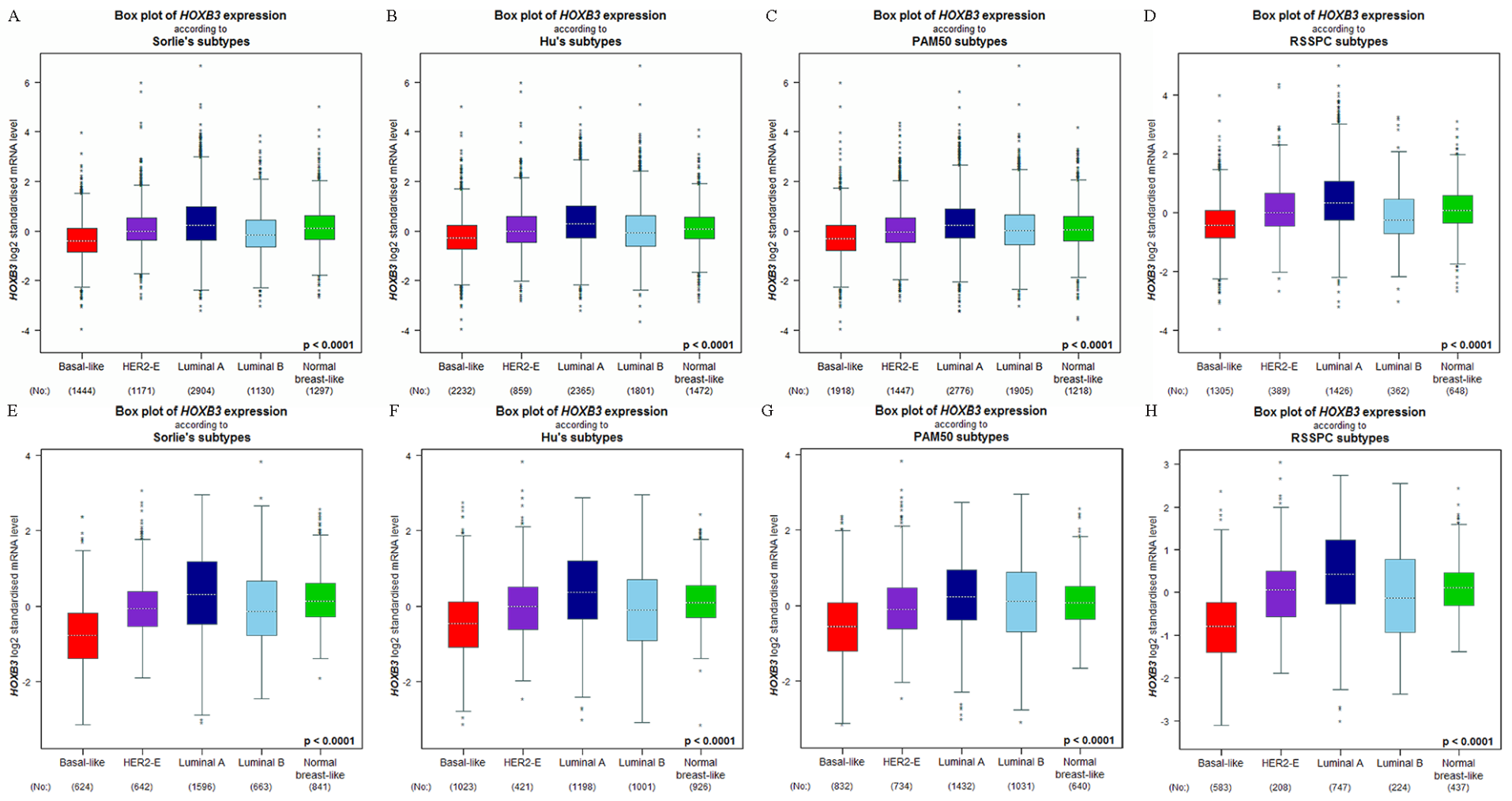

Figure 5

The relationship between HOXB3 and different molecular subtypes in breast cancer. (A-D) Box plots of HOXB3 expression according to different molecular subtypes (in DNA microarrays' level). (E-H) Box plots of HOXB3 expression according to different molecular subtypes (in RNA-sequence level). Global significant differences between groups were assessed by Welch's test, and $\mathrm{P}<0.05$ was considered statistically significant. 
A

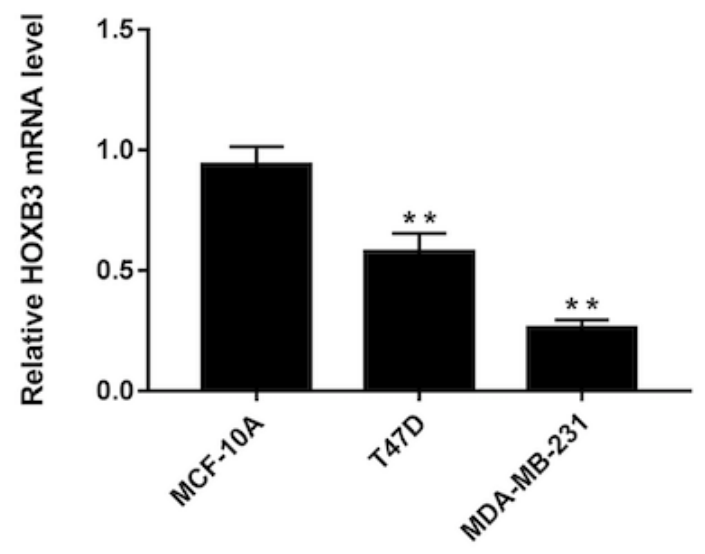

C

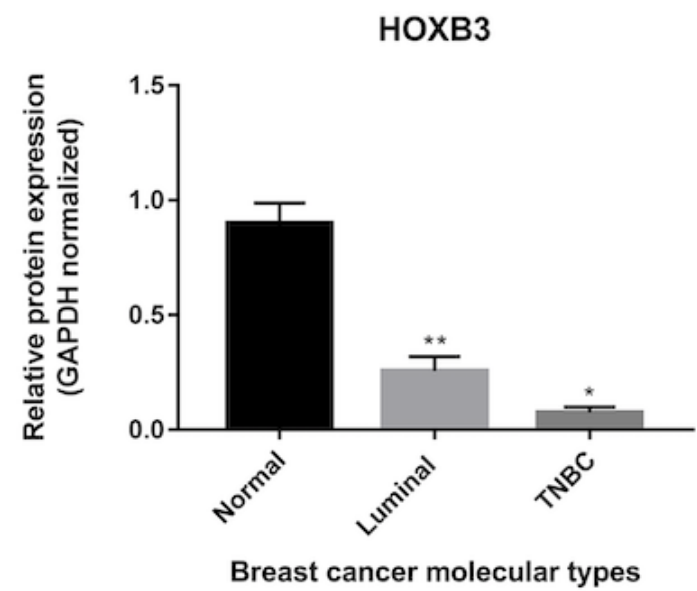

B
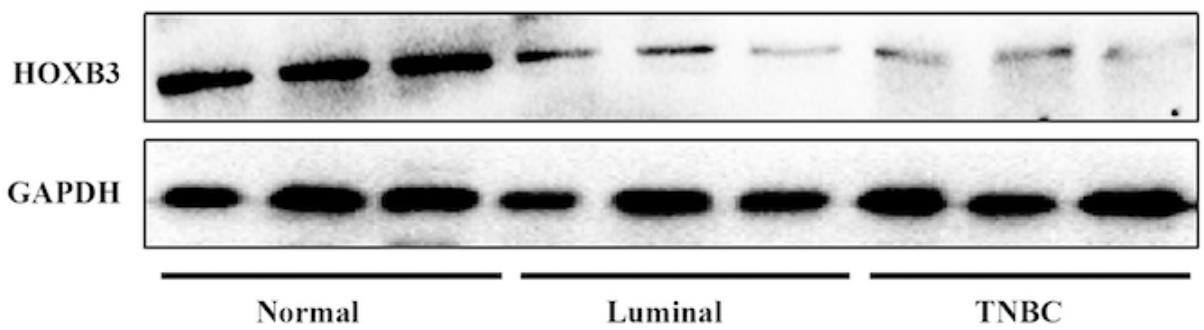

D
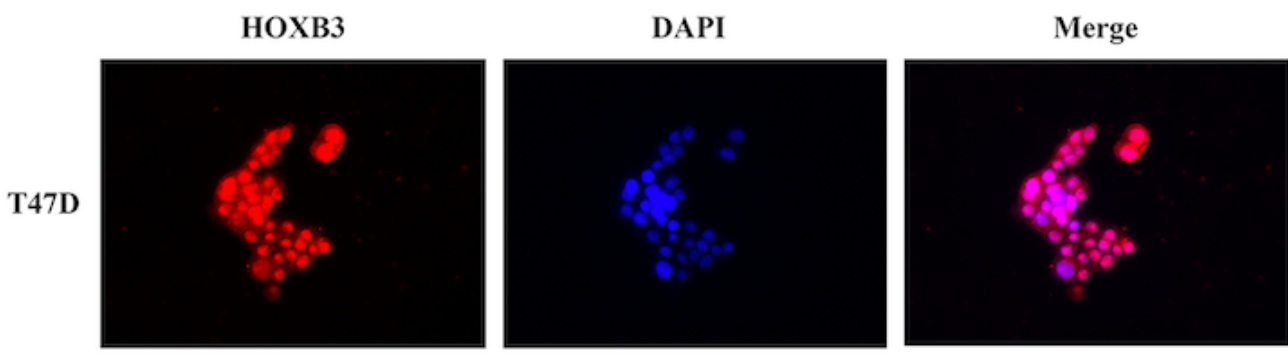

MDA-MB-231
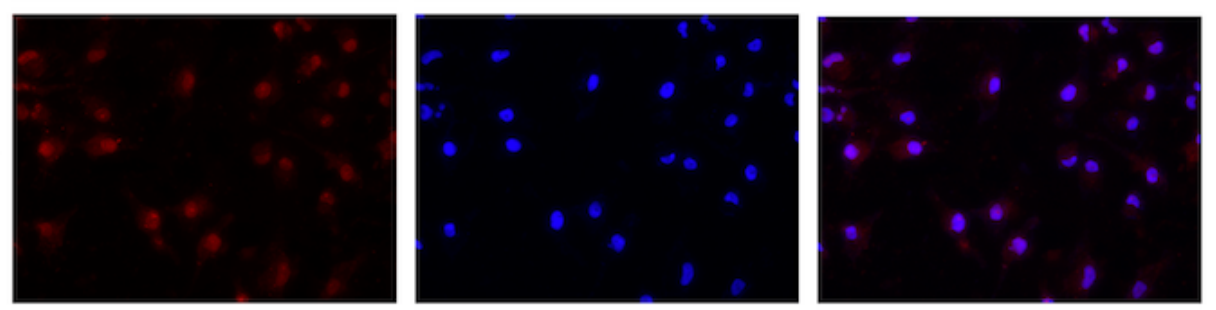

\section{Figure 6}

HOXB3 expression in breast cancer cell lines and breast cancer tissues (A) The relative HOXB3 mRNA level of MCF-10A, T47D and MDA-MB-231 by qRT-PCR. (B) Western blot of HOXB3 expression in normal breast tissues, luminal breast cancer and TNBC. (C) The relative protein expression of HOXB3 (GAPDH

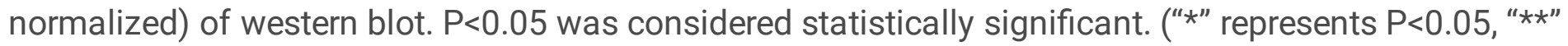
represents $\mathrm{P}<0.01$ ) (D) Immunofluorescence of HOXB3 expression in T47D and MDA-MB-231. 


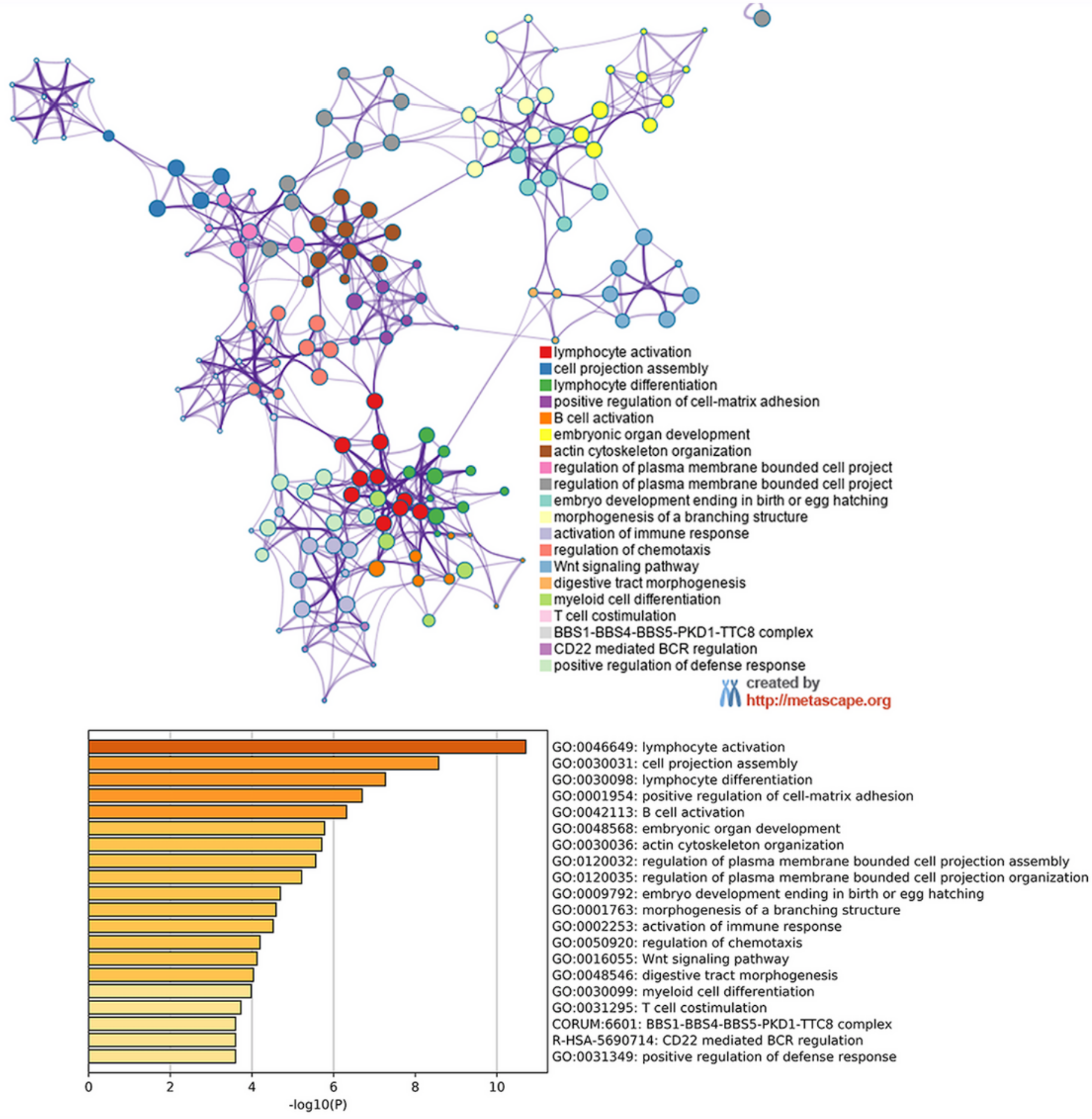

Figure 7

Metascape for GO analysis of possible mechanism of HOXB3 in breast cancer.

\section{Supplementary Files}

This is a list of supplementary files associated with this preprint. Click to download. 
- supplementaryfigure1.JPG

Page 18/18 